\title{
OSCILLATION CRITERIA FOR CERTAIN EVEN ORDER DIFFERENTIAL EQUATIONS WITH DISTRIBUTED DEVIATING ARGUMENTS
}

\section{GEFENG YANG AND Zhiting XU}

Abstract. By using averaging function and the approach developed by Philos and Kong, Kamenevtype and interval oscillation criteria are established for the even order differential equation with distributed deviating arguments,

$$
\left(r(t)\left|x^{(n-1)}(t)\right|^{p-1} x^{(n-1)}(t)\right)^{\prime}+\int_{\alpha}^{\beta} F[t, \xi, x(g(t, \xi))] d \sigma(\xi)=0 .
$$

The obtained results are extensions of existing ones for second order linear differential equations.

Mathematics subject classification (2010): 34C10, 34C15.

Keywords and phrases: oscillation, differential equations, distributed deviating arguments, even order.

\section{REFERENCES}

[1] R.P. Agarwal, S.R. GRACE, D. O’Regan, Oscillation criteria for certain $n$-th order differential equation with deviating arguments, J. Math. Anal. Appl., 262 (2001), 601-622.

[2] R.P. AgARWAL, S.R. GRACE, Oscillation theorems for certain functional differential equation of higher order, Math. Comput. Modelling., 39 (2004), 1185-1194.

[3] R.P. Agarwal, S.R. Grace, I. Kiguradze, D. O’Regan, Oscillation of functional differential equations, Math. Comput. Modelling., 41 (2005), 417-461.

[4] S.R. GRACE, B.S. LALLI, Oscillation theorems for $n$th order delay differential equations, J. Math. Anal. Appl., 91 (1983), 352-366.

[5] S.R. GRACE, B.S. LALLI, Oscillation theorems for damping differential equations of even order with deviating arguments, SIAM. J. Math. Anal., 15 (1984), 308-316.

[6] G.H. Hardy, J.E. Littlewood, G.Pölya, Inequalities, 2nd edition, Cambridge University Press, 1988.

[7] I.V. Kamenev, An integral criterion for oscillation of linear differential equations, Mat. Zametki., 23 (1978), 249-251.

[8] I.T. KigURADZE, On the oscillation of solutions of equation $\frac{d^{m} u}{d t^{m}}+a(t) u^{m}$ signu $=0$, Mat. Sbornik., 65 (1964), 172-187.

[9] Q. Kong, Interval criterion for oscillation of second order linear differential equation, J. Math. Anal. Appl., 229 (1999), 258-270.

[10] R.G. KoplatADZE, On oscillatory properties of solutions of functional differential equations, Mem. Differential Equations Math. Phys., 3 (1994), 1-179.

[11] CH.G. Philos, Oscillation theorems for linear differential equations of second order, Arch. Math(Basel)., 53 (1989), 482-492.

[12] S. RuAn, Oscillations of $n$-th order functional differential equations, Comput. Math. Appl., 21 (1991), 95-102.

[13] Y. TANG, Q. YANG, Oscillation of even order nonlinear functional differential equations with damping, Acta Math. Hungar., (3), 102 (2004), 223-238. 
[14] A. TRIYAKI, Y. BASCI, I. GÜLEC, Interval criteria for oscillation of second order functional differential equations, Comput. Math. Appl., 50 (2005), 1487-1498.

[15] P. WANG, J. ZHANG, Oscillatory criteria for even order half-linear differential equations with distributed deviating arguments, Commun. Appl. Anal., 2-3, 10 (2006), 329-342.

[16] J.S.W. Wong, On Kamenev-type oscillation theorems for second-order differential equations with damping, J. Math. Anal. Appl., 258 (2001), 244-257.

[17] H.W. WU, Q.R. WANG, Y.T. XU, Oscillation criteria for certain even order nonlinear functional differential equations, Dynam. Systems Appl., 13 (2004), 127-142.

[18] Z. XU, Y. XIA, Integral averaging technique and oscillation of certain even order delay differential equations, J. Math. Anal. Appl., 292 (2004), 238-246.

[19] S. Zhang, F. MENG, Oscillation criteria for even order neutral equations with distributed deviating arguments, Int. J. Differ. Equ. Vol., 2010, Article ID 308357, 14 pages. 